\title{
Characterization of a plasma window as a membrane free transition between vacuum and high pressure
}

\author{
B. F. Bohlender, ${ }^{*}$ A. Michel, ${ }^{\dagger}$ J. Jacoby, and M. Iberler® \\ Goethe Universität Frankfurt, Max-von-Laue-Str. 1, 60438 Frankfurt am Main \\ O. Kester@ \\ TRIUMF, 4004 Wesbrook Mall, Vancouver, BC V6T 2A3, Canada
}

(Received 19 November 2019; published 15 January 2020)

\begin{abstract}
A plasma window (PW) is a device for separating two areas of different pressures while letting particle beams pass with little to no loss. It has been introduced by A. Hershcovitch [J. Appl. Phys. 78, 5283 (1995)] In the course of this publication, the properties of a PW with apertures of $3.3 \mathrm{~mm}$ and $5.0 \mathrm{~mm}$ are presented. Especially the link between the pressure properties relevant for applications in accelerator systems and the underlying plasma properties depending on external parameters are presented. As working gas, a $98 \% \mathrm{Ar}-2 \% \mathrm{H}_{2}$ mixture has been used due to the intense Stark broadening of the $\mathrm{H}_{\beta}$-line and the welldescribed Ar characteristics, enabling an accurate electron density and temperature analysis. At the low pressure side around some mbar, high-pressure values reached up to 750 mbar while operating with volume flows between $1 \mathrm{slm}$ and $4 \mathrm{slm}$ (standard liter per minute) and discharge currents ranging from 45 A to $60 \mathrm{~A}$. The achieved ratios between high and low pressure with an active discharge range from 40 to 150. This is an improvement of a factor up to 12 over the performance of an ordinary differential pumping stage of the same geometry. Unique features of the presented PW include simultaneous plasma parameter determination and the absence of ceramic insulators between the cooling plates. Optical analysis reveals no significant damage or wear to the components after an operation time well over $10 \mathrm{~h}$, whereas the cathode needle needs replacement after $5 \mathrm{~h}$.
\end{abstract}

DOI: 10.1103/PhysRevAccelBeams.23.013501

\section{INTRODUCTION}

Modern atomic and particle physics call for intense and brilliant particle beams with high energies, while specific applications require the separation between the accelerator's vacuum and areas of higher pressures, e.g., gas strippers or experimental chambers. Especially when producing radioactive isotopes in the course of an experiment, the accelerator needs to be shielded from debris to prevent contamination.

Technological applications aside from the usage in accelerator systems include atmospheric electron welding, cutting and surface modification also calling for a reliable vacuum-atmosphere interface. Additionally, x-ray microscopy in living cells suffers from the degrading of the used SiN-Windows and their small size, again demanding a long living interface.

\footnotetext{
*bohlender@iap.uni-frankfurt.de

Also at GSI Helmholtzzentrum fr Schwerionenforschung.
}

Published by the American Physical Society under the terms of the Creative Commons Attribution 4.0 International license. Further distribution of this work must maintain attribution to the author(s) and the published article's title, journal citation, and DOI.
Conventional means of beam transfer from vacuum to regions of high pressure is usually archived by using metallic membranes or differential pumping stages. Growing beam intensities limit the usage of membrane windows due to their destruction by beam interaction, while differential pumping stages grow unacceptable long for high pressures. Therefore, the physical community seeks for material free separation of high pressure regions in accelerators.

A possible alternative has been proposed by A. Hershcovitch in 1995 [1]: The improvement of a differential pumping stage by introducing an arc discharge into the stage. This so-called plasma window (PW) uses a cascaded arc discharge [2] for the connection between vacuum and high pressure. The plasma is ignited in a channel connecting the different pressure areas formed by insulated copper discs. These discs stabilize the arc due to spatial and thermal confinement and need to be water cooled due to the high power dissipation, $P_{\text {loss }} \leq 1 \mathrm{k} \mathrm{W} \mathrm{cm}^{-1}$, and temperature $T \simeq 1 \times 10^{4} \mathrm{~K}$ of the discharge [2].

While the pressure ratio characteristics of the PW have been subject to research and simulations for apertures between $2 \mathrm{~mm}$ and $8 \mathrm{~mm}$ [1,3-7], the underlying plasma properties have only sparsely been published, one exception 


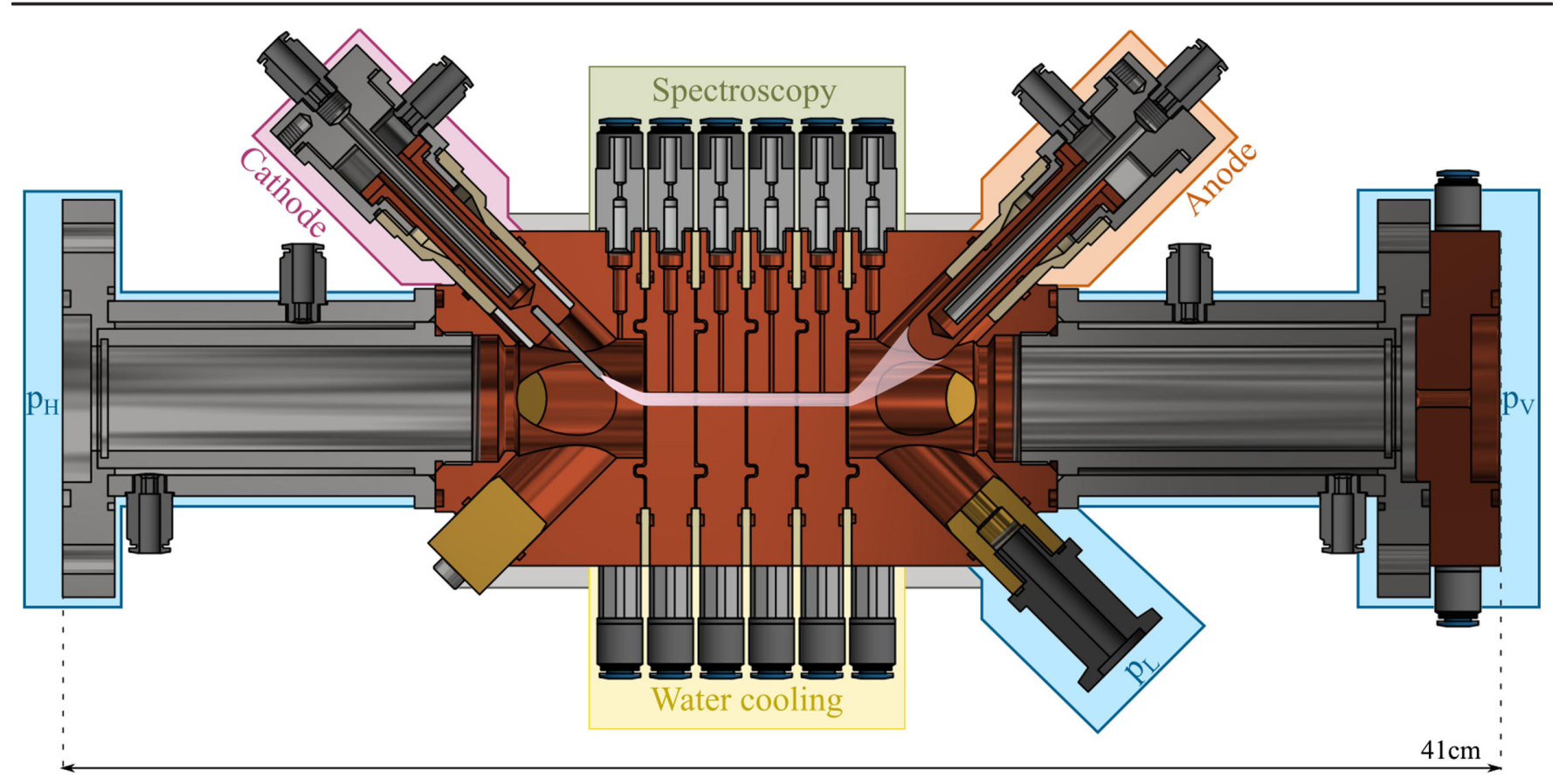

FIG. 1. Cross section of the PW in use, the geometrical parameters are given in Table I. The high pressure side $p_{H}$ is located to the left. The active discharge is indicated as a bright pink area.

being [8] for the case of a hydrogen discharge. For future applications and development of the plasma window technology, insight into the linking of external parameters to the inner plasma characteristics and the achievable pressure differences needs to be researched. Especially for the development of large aperture PWs an understanding of the working mechanisms is necessary.

Transmission of particles through a PW has been shown for electrons, vacuum ultraviolet and soft x-rays, although these publications [1,9-12] only state particles or photons were transmitted through the PW. Studies on the transmission characteristics or the influence of the plasma on particle beam properties like average charge state or emittance have not been conducted and/or published yet.

This paper presents the basic gas dynamic and plasma properties inside the $\mathrm{PW}$ with apertures of $3.3 \mathrm{~mm}$ and $5.0 \mathrm{~mm}$, the plasma properties and their influence on the achievable pressure ratios of the PW.

Of particular interest for further applications of the plasma window technology is the improvement of the pressure ratio in relation to a differential pumping stage. The improvement in sealing of the plasma window compared to a differential pumping stage can be expressed as

$$
q_{n}=\frac{p_{H}}{p_{H, 0}}
$$

where $p_{H}$ is the high side pressure with active discharge and $p_{H, 0}$ is that of a conventional pumping stage with the same geometric properties as the PW at fixed particle flow. $q_{n}$ will later on be used to classify the performance of the presented PW.

\section{A. Plasma physics}

As the PW's sealing mechanism is supposed to originate in the heating of the working gas [1], a look into the thermodynamic properties of plasmas is worthwhile.

A plasma is composed of different particle species, usually electrons, ions, and neutral atoms or molecules. Generally speaking, the electrons usually carry significant higher energies than the heavier species. If all species carry locally the same kinetic energy, the plasma is in a state called local thermodynamic equilibrium (LTE).

For a LTE to prevail, the electron density $n_{e}$ needs to be high enough to ensure sufficient energy transfer from the fast and light electrons to the heavier species. [13] formulated an expression to calculate the necessary electron density:

$$
\frac{n_{\text {crit }}}{\mathrm{cm}^{-3}}=1 \times 10^{14}\left(\frac{k_{B} T_{e}}{\mathrm{eV}}\right)^{3}\left(\frac{\epsilon_{\text {ion }}}{k_{B} T_{e}}\right)^{5 / 2},
$$

where $k_{B} T_{e}$ is the mean kinetic energy of the electrons, $\epsilon_{\text {ion }}$ is the ionization energy of the species under question.

For an Argon plasma with electron temperatures around $k_{B} T_{e} \approx 1 \mathrm{eV}$, this yields a critical electron density around $n_{\text {crit }}=9.9 \times 10^{16} \mathrm{~cm}^{-3}$.

It is worthwhile to stress that only if the observed electron density is higher than $n_{\text {crit }}$, the heavy particle temperature is equivalent to that of the electrons. Otherwise, no accurate determination of the heavy particle temperature is possible, but it increases with increasing electron density. 


\section{EXPERIMENTAL SETUP AND DATA EVALUATION}

\section{A. Experimental setup}

Figure 1 shows the cross section of the PW developed at IAP. On the left side of the schematic, the recipient containing the high pressure $p_{H}>100$ mbar is shown. The PW itself consists of the cathode section upstream, the anode section downstream of the plasma and four copper plates connecting cathode and anode section. The cathode, formed from a W- $\mathrm{La}_{2} \mathrm{O}_{3}$ pin, is being held by a water cooled copper body which is insulated by a PEEK-sleeve and MACOR hood from the body of the setup. The anode is located at the lower pressure side and made from copper.

The cooling plates are made from copper and separated by PEEK-Spacers. These spacers usually suffer damage from the high intensity optical radiation emitted by the plasma and need to be shielded from this radiation. Designs by other groups achieve this by using boron nitride washers, while the here presented PW implements a tongue-and-groove design, which is less prone to failure and easier to manufacture than brittle ceramics.

Due to the spacial confinement of the arc, the PEEK insulators have no contact with the plasma and prove to be durable. On the other hand, the MACOR hood suffers from arcing with the ignition pulse, so that another choice of material will be necessary in the future. Once ignited, the discharge anchors at the cathode tip, so that the MACOR does not suffer too much damage.

For operation of the PW, a gas mixture of $98 \% \mathrm{Ar}+2 \%$ $\mathrm{H}_{2}$ is fed into the setup at several flow rates in the range from $1 \mathrm{slm}$ up to $4 \mathrm{slm}$. This is done to enable an accurate temperature determination via Boltzmannplot technique using up to 11 ArII-Lines, while the $\mathrm{H}_{2}$-addition allows for precise electron density calculation. In order to record the necessary spectra, each cooling plate features an optical port. This allows the simultaneous acquisition of spectral data emerging from different points along the discharge axis at the same time. Combined, these measurements allow for insight into the plasma characteristics under different global parameter settings, such as current, pressure and volume flow rate.

Table I summarizes the experimental parameters under which the presented measurements have been conducted. The data presented in this contribution was collected by using a scroll pump at the low pressure side.

TABLE I. Geometry and setup data for the IAP PW.

\begin{tabular}{lr}
\hline \hline Length over all & $41 \mathrm{~cm}$ \\
Channel length & $59 \mathrm{~mm}$ \\
Channel diameter & 3.3 and $5.0 \mathrm{~mm}$ \\
Current & $40 \ldots 60 \mathrm{~A}=$ \\
Voltage & $\leq 150 \mathrm{~V}_{=}$ \\
Gas mixture & $\mathrm{Ar}+2 \% \mathrm{H}_{2}$ \\
Pumping speed & $\approx 400 \mathrm{slm}$ \\
\hline \hline
\end{tabular}

\section{B. Electron density and temperature evaluation}

Plasma characterization is done by spectroscopic methods. For the calculation of the electron density $n_{e}$ from the $\mathrm{H}_{\beta}$-broadening, the following formula from [14] is used:

$$
\mathrm{FWHM}=2 \gamma=4.8 \mathrm{~nm}\left(\frac{n_{e}}{1 \times 10^{17} \mathrm{~cm}^{-3}}\right)^{0.68116}
$$

In Eq. (3) FWHM is the full width at half maximum of the broadened line profile. The accuracy of the measured electron density is typically better than $10 \%$.

As for the electron temperature determination, the Boltzmannplot [15] method with selected ArII-lines is used. The used lines and some of their relevant quantities are given in Table II. In order to achieve a good temperature estimation, spectra with three different wavelength frames were recorded. The optical setup's response was adjusted for its sensitivity in the full range of the observed lines. By doing so, up to 11 ArII-lines could be used for the electron temperature determination at any given set of parameters and optical ports, resulting in an accuracy around $7.5 \%$.

The used spectra were recorded through radial windows in the PW, see Fig. 1, and a 0.5 m-Monochromator.

\section{Acquisition of electrical and pressure data}

The discharge current and voltage was recorded from the main power supply. This is done since the available oscilloscopes are used to record the potential distribution when igniting the plasma. For the future, direct voltage measurements across the window itself will be conducted. The voltage drop across the used Diode, $U_{\text {Diode }}=1 \mathrm{~V}$ has been subtracted from the voltage indicated by the power supply. The power supply has an accuracy of $\Delta U=1.25 \mathrm{~V}$ and $\Delta I=0.3 \mathrm{~A}$. Since no additional ballast resistor or inductor is used, the voltage at the power supply minus $U_{\text {Diode }}$ equals the voltage across the discharge.

TABLE II. ArII-lines used for the temperature determination via Boltzmann plot technique, taken from [16].

\begin{tabular}{lcrrll}
\hline \hline$\lambda / \mathrm{nm}$ & \multicolumn{1}{c}{$A / \mathrm{s}^{-1}$} & $g_{p}$ & $E_{p} / \mathrm{eV}$ & Configuration & Term \\
\hline 457.93 & $8.0 \times 10^{7}$ & 2 & 19.973 & $3 s^{2} 3 p^{4}\left({ }^{3} P\right) 4 p$ & ${ }^{2} S_{1 / 2}^{\circ}$ \\
458.98 & $6.64 \times 10^{7}$ & 6 & 21.127 & $3 s^{2} 3 p^{4}\left({ }^{1} D\right) 4 p$ & ${ }^{2} F_{5 / 2}^{\circ}$ \\
460.96 & $7.89 \times 10^{7}$ & 8 & 21.143 & $3 s^{2} 3 p^{4}\left({ }^{1} D\right) 4 p$ & ${ }^{2} F_{7 / 2}^{\circ}$ \\
465.79 & $8.92 \times 10^{7}$ & 2 & 19.801 & $3 s^{2} 3 p^{4}\left({ }^{3} P\right) 4 p$ & ${ }^{4} P_{1 / 2}^{\circ}$ \\
480.60 & $7.80 \times 10^{7}$ & 6 & 19.223 & $3 s^{2} 3 p^{4}\left({ }^{3} P\right) 4 p$ & ${ }^{4} P_{5 / 2}^{\circ}$ \\
484.78 & $8.49 \times 10^{7}$ & 2 & 19.305 & $3 s^{2} 3 p^{4}\left({ }^{3} P\right) 4 p$ & ${ }^{4} P_{1 / 2}^{\circ}$ \\
487.98 & $8.23 \times 10^{7}$ & 6 & 19.680 & $3 s^{2} 3 p^{4}\left({ }^{3} P\right) 4 p$ & ${ }^{2} D_{5 / 2}^{\circ}$ \\
496.50 & $3.94 \times 10^{7}$ & 4 & 19.762 & $3 s^{2} 3 p^{4}\left({ }^{3} P\right) 4 p$ & ${ }^{2} D_{3 / 2}^{\circ}$ \\
497.21 & $9.7 \times 10^{6}$ & 2 & 19.305 & $3 s^{2} 3 p^{4}\left({ }^{3} P\right) 4 p$ & ${ }^{4} P_{1 / 2}^{\circ}$ \\
500.93 & $1.51 \times 10^{7}$ & 6 & 19.223 & $3 s^{2} 3 p^{4}\left({ }^{3} P\right) 4 p$ & ${ }^{4} P_{5 / 2}^{\circ}$ \\
501.71 & $2.07 \times 10^{7}$ & 6 & 21.127 & $3 s^{2} 3 p^{4}\left({ }^{1} D\right) 4 p$ & ${ }^{2} F_{5 / 2}^{\circ}$ \\
\hline \hline
\end{tabular}




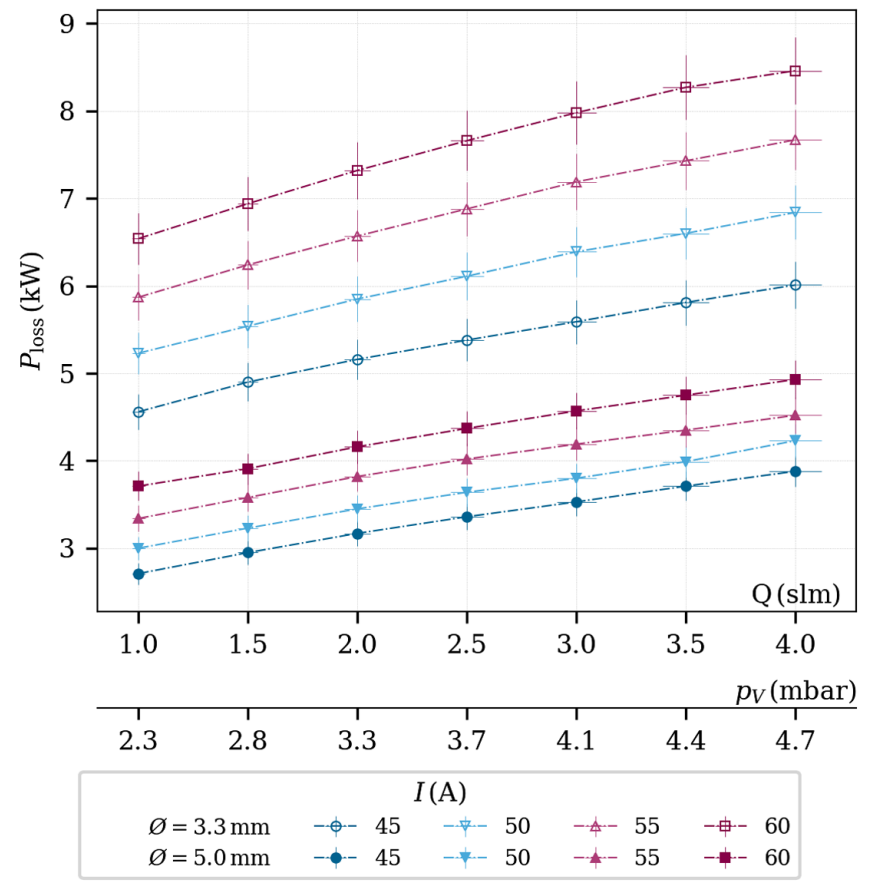

FIG. 2. Electrical power $P_{\text {loss }}$ in dependence of current and pressure. Error bars in x-direction refer to the error in $Q$.

Pressure values at the cathode and anode, see $p_{H}$ and $p_{L}$ in Fig. 1, were taken with two Agilent PCG-750 manometers. The pressure in the pumping recipient, $p_{V}$ in Fig. 1, was recorded using a Pfeiffer PKR 251 manometer. The used manometers have typical errors of $5 \%$ (Agilent) and $30 \%$ (Pfeiffer) respectively.

\section{RESULTS AND DISCUSSION}

\section{A. Electrical parameters}

The electrical measurements show that the power needed for sustaining the discharge scales with pressure and current but drops with increasing aperture. The dependence is shown in Fig. 2.

The increase of $P_{\text {loss }}$ with increasing current and pressure and decreasing aperture originates from the increase of neutral particles within the channel, see Sec. III D and [17]. As a consequence, the plasma's electrical resistance grows [18], therefore a higher voltage is necessary to sustain the discharge the same current.

\section{B. Plasma parameters}

The recorded spectra are used to calculate the electron density $n_{e}$ and temperature $k_{B} T_{e}$ at every observation point. Calculations are done according to the description given in Sec. II B. These calculated values are averaged over the discharge axis and shown in Figs. 3 and 4 as $\left\langle n_{e}\right\rangle$ and $\left\langle k_{B} T_{e}\right\rangle$ to illustrate the collective behavior of these quantities.

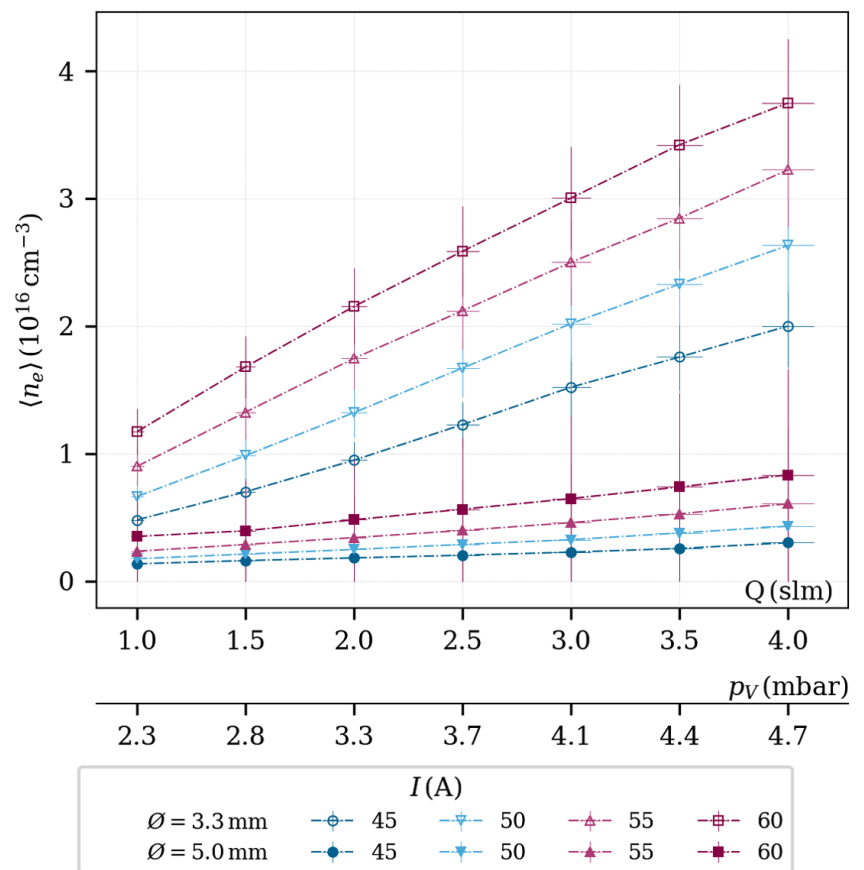

FIG. 3. Electron density $\left\langle n_{e}\right\rangle$ averaged along discharge channel in dependence of current and pressure. Error bars in X-direction refer to the error in $Q$.

Electron density $\left\langle n_{e}\right\rangle$ scales with current and volume flow, which is in good agreement with [19] and [20]. The increase of $\left\langle n_{e}\right\rangle$ with the volume flow originates from the higher number of particles inside the plasma and indicates a constant degree of ionization. Due to the limited discharge

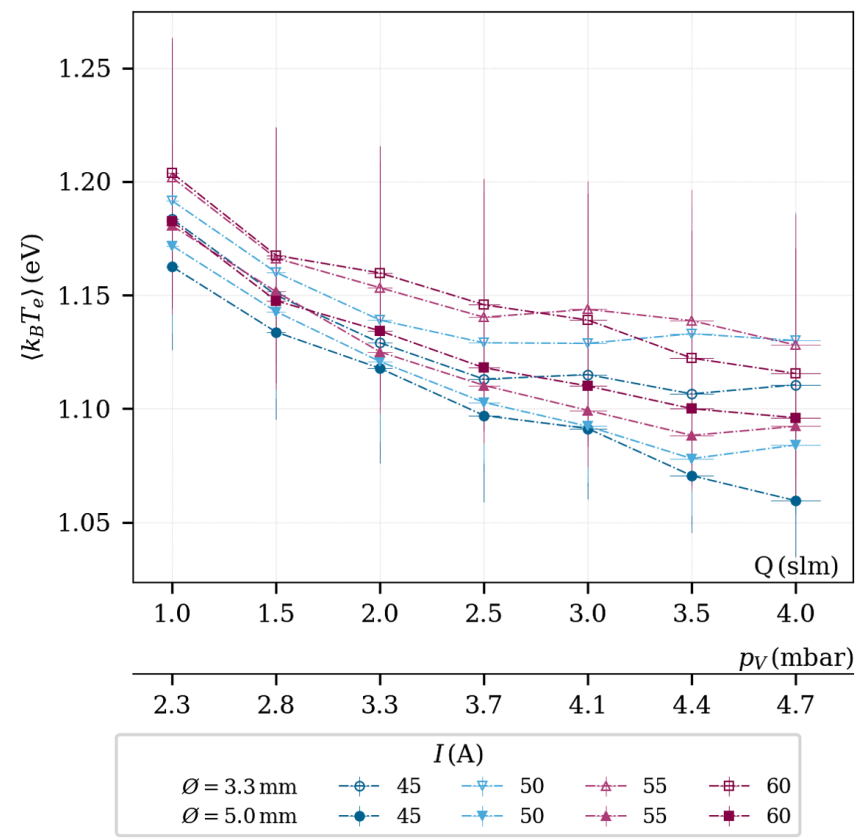

FIG. 4. Electron temperature $\left\langle k_{B} T_{e}\right\rangle$ averaged along discharge channel in dependence of current and pressure. Error bars in x-direction refer to the error in $Q$. 


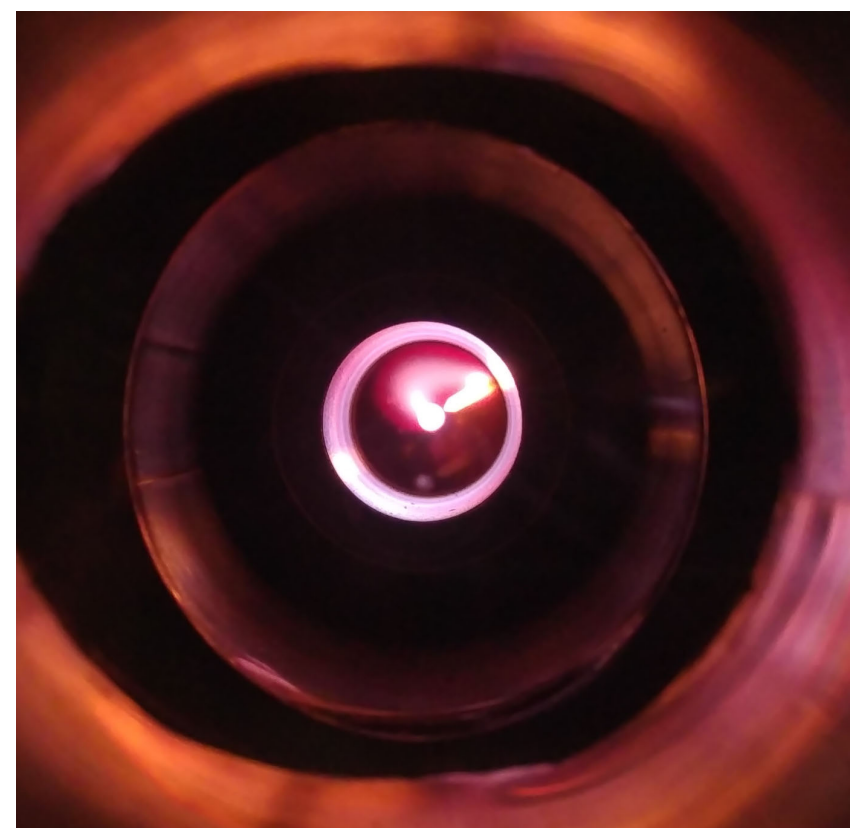

FIG. 5. View onto the discharge from the cathode side. The plasma filled discharge channel is the bright central circle, the cathode sticks out from the upper right side. Visible is the anchor point which has already moved up the tip.

cross section, an increase of the current gives rise to a higher current density, thus a higher number of electrons inside the discharge.

The maximal density is $3.75 \times 10^{16} \mathrm{~cm}^{-3}$, which is not sufficient for the plasma to be in a LTE, see Sec. I A. Therefore, no valid statement can be made about the heavy particle temperature, but according to [17] and [19], the heavy particle temperature increases with increasing electron density.

The electron temperature $\left\langle k_{B} T_{e}\right\rangle$ is decreasing with increasing particle flow. With more particles inside the plasma volume, the collision frequency increases, thus electrons transfer more energy to heavy particles, reducing the electron temperature [19]. On the contrary, an increase of the discharge current seems to have no significant effect on the electron temperature.

\section{Lifetime}

Optical investigation of the cooling plates and the anode reveals no significant damage after well over $10 \mathrm{~h}$ of operation.

In contrast to this stability, the discharge's anchor point at the cathode tip drifts toward the cathode body, as indicated in Fig. 5. The drift begins to become visible after about $1 \mathrm{~h}$ of operation. Since the cathode's body is made from copper, the tip needs to be replaced before the discharge reaches the body, which would inevitably melt otherwise. Due to the needed replacement, the operation time is limited to $5 \mathrm{~h}$ at a stretch.
Our assumption about the cause of this drift is based on the tip's material. Due to the high temperature of the needle under operation, the Lanthanoxide may burn out from the tip, leaving pure tungsten material. Since Tungsten has a higher work function than the Tungsten-Lanthanoxide compound, the anchor drifts away from the burnt out areas.

A possible enhancement of the lifetime might thus arise from using pure Tungsten tips in the future, which comes with the drawback of the then needed increased electrical power to sustain and ignite the discharge. To encounter the harder ignition, the development of heated cathodes is at work, yielding promising first results. Since thoriated Tungsten has a significant lower work function than pure Tungsten, it is easier to ignite, however due to safety regulations, the use of those is not possible in our lab.

Another possibility to improve the cathode's lifetime is the use of multiple cathodes simultaneously. This will be tested once the heated cathode tests are completed.

\section{Pressure parameters}

Considering the particle flow $\Gamma=Q \times n$ and using Hagen-Poiseuille's law for compressible fluids as an approximation for the gas flow throughout the window, one can derive

$$
\Gamma=\frac{\pi R^{4}}{32 l}\left(\frac{p_{H}^{2}-p_{V}^{2}}{p_{V}}\right) \frac{n}{\eta}
$$

where $l$ is the length of the channel, $R$ its radius, $n$ the particle density, and $\eta$ the viscosity of the fluid under consideration.

Rearranging Eq. (4) under the assumption that $p_{H}^{2} \gg p_{V}^{2}$, an expression for $p_{H}$ can be formulated:

$$
p_{H} \approx \frac{1}{\pi R^{2}} \sqrt{32 l \Gamma \pi p_{V}} \sqrt{\frac{\eta}{n}} .
$$

Since the viscosity of a gas increases with its temperature while its density decreases [18], an increase of the gas temperature induces higher value for $p_{H}$, if the particle flow and thus $p_{V}$ are kept constant.

The scaling of high side pressure $p_{H}$ with applied volume flow $Q_{0}$ and discharge current $I$ is depicted in Fig. 6. Clearly the channel's aperture has the largest influence on the obtained high pressure $p_{H}$. The shown weak scaling of $p_{H}$ with $I$ is in good agreement with the data published in [3] for currents above $10 \mathrm{~A}$.

As the viscosity of gases increases with increasing temperature at most by a factor 3 [18], the viscosity's influence on the performance of the PW is limited.

The ratio $q_{n}=\frac{p_{H}}{p_{H .0}}$ characterizes the sealing improvement of the PW over the same setup operated as an ordinary differential pumping stage. This quantity could be used in future publications for comparing different $\mathrm{PW}$ from 


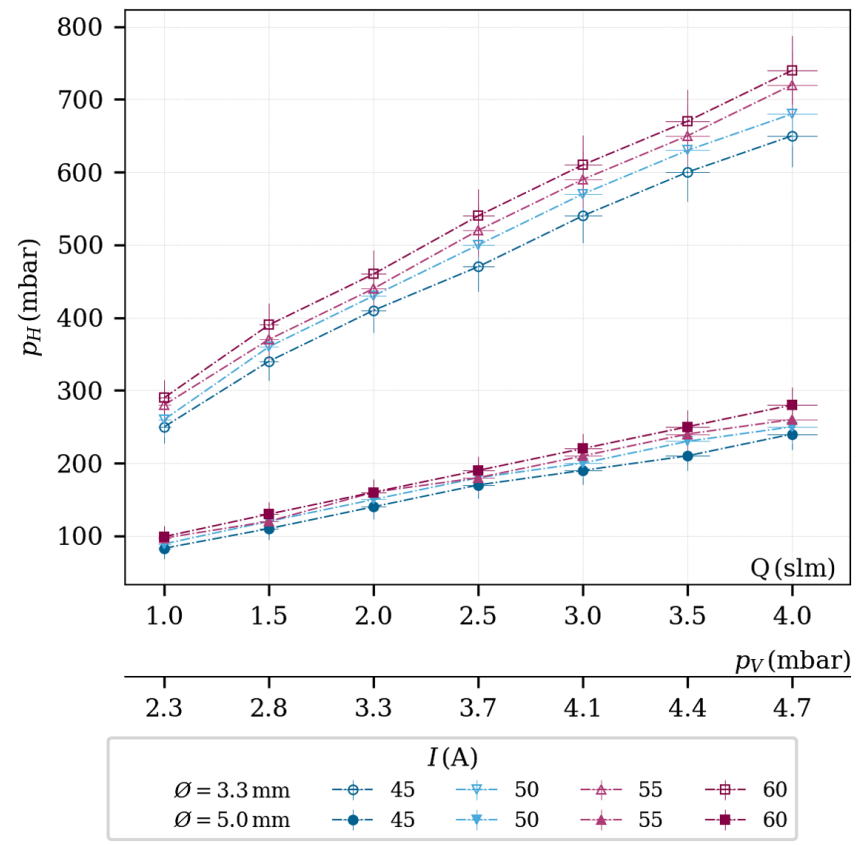

FIG. 6. High pressure $p_{H}$ in dependence of the discharge current $I$ and volume flow $Q$. Error bars in x-direction refer to the error in $Q$, the systematic error in $p_{V}$ is $30 \%$.

varying groups in terms of their performance. Its behavior with external parameters is depicted in Fig. 7.

An improvement of a factor up to 12 can be seen from the data presented in Fig. 7. As with high pressure, the smaller channel diameter yields a better performance.

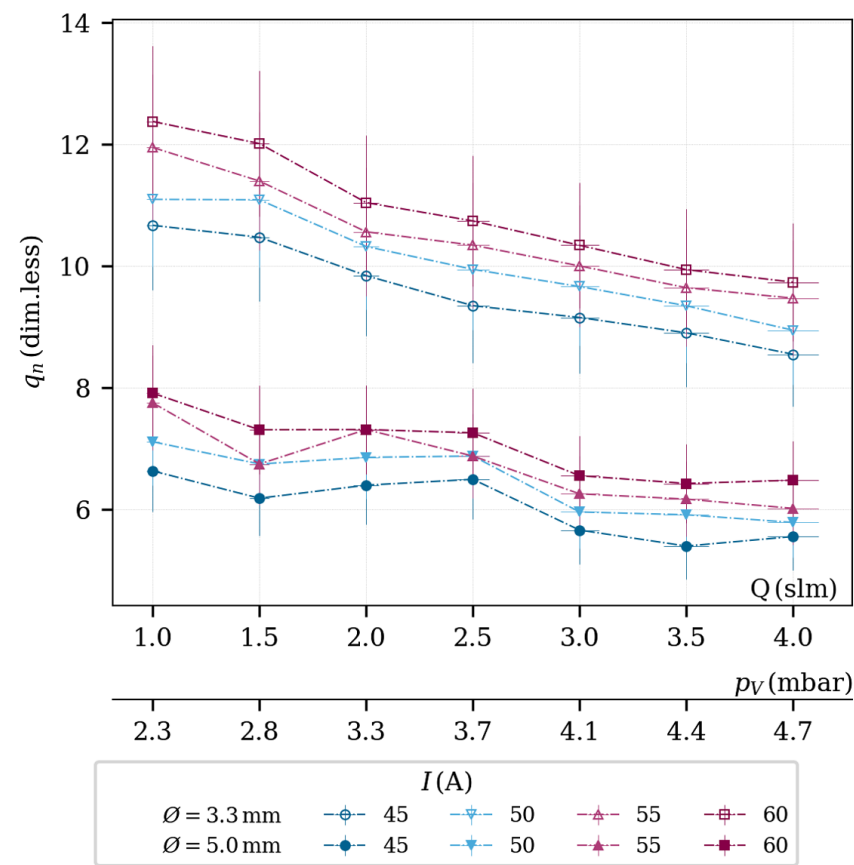

FIG. 7. Normalized pressure quotient $q_{n}=\frac{q}{q_{0}}$ in dependence of discharge current $I$ and volume flow $Q$. Error bars in x-direction refer to the error in $Q$.
For both used diameters $q_{n}$ scales with the current and against the volume flow.

\section{CONCLUSION}

It has been shown that the PW developed at IAP is capable of maintaining a pressure difference up to $p_{H}=$ 750 mbar to $p_{V}=4.7 \mathrm{mbar}$ for over $5 \mathrm{~h}$ at continuous operation with little to no signs of erosion. This is achieved using a single scroll pump with a pumping speed of $200 \mathrm{slm}$. The sealing is caused by increasing the heavy particle temperature so that the particle density inside the channel decreases and the viscosity of the gas increases. Heating of the particles is achieved by increasing the electron density, which causes a more efficient energy transfer from the electrons to the heavy particles.

The achievable pressure ratio originates mostly from the decrease of particle density inside the channel III D, thus a higher bulk temperature is needed for further performance improvement. Theoretically, a bulk temperature as high as possible would yield the best performance results. However, the bulk temperature cannot exceed the electron temperature which in turn is limited to values around $0.8 \mathrm{eV}$ to $1.2 \mathrm{eV}$ for $\mathrm{Ar}-\mathrm{H}_{2}$ mixtures at atmospheric pressure and sustainable DC-currents [21]. Once the maximum temperature is reached, no increase in performance due to the decrease of the particle density inside the channel is to be expected.

The water cooling of the downstream recipient proved crucial, as the hot gas streaming out of the discharge heats the recipient up to a point where the sealing starts to fail. For prolonged operation of the window, other cathode materials than $\mathrm{W}-\mathrm{La}_{2} \mathrm{O}_{3}$ need to be tested since this compound proved not to be stable enough of long term operation. Additionally, using heated cathodes or multiple cathodes (the setup allows for up to four) at once are under investigation.

In order to characterize the PW's performance, additional tests with different working gases are scheduled for the near future. Additionally, the test of pure tungsten cathode pins and studies of the influence of the number of cathodes on the PW's lifetime are on their way. Furthermore, an upgrade of the vacuum system will be performed, presumably increasing the achievable pressure difference.

For the future, beam interaction tests are planned to specify the transmission properties of the PW for different particles. As the presented data indicates that the achievable pressure ratio decreases with increasing channel aperture. In order to encounter this phenomenon, either a significant increase of the discharge current, a higher volume flow or an enhancement of the experimental technique are necessary. Since the current and volume flow cannot be increased ad infinitum, the experimental technique needs to be reflected and enhanced. 
A possible enhancement of the setup for larger apertures is based on an superimposed axial magnetic field with an appropriate strength. Electrons within the plasma will gyrate around the magnetic field lines, thus the length they travel within the plasma increases. As a result, the number of collisions between electrons and ions or atoms increases, leading to a higher bulk temperature, again decreasing the particle density inside the channel.

\section{ACKNOWLEDGMENTS}

The here presented work would not have been possible without the support of the Bundesministerium für Bildung und Forschung (BMBF), Reference No. 05P15 RFRBA, Helmholtz International Center (HIC) for FAIR (Facility for Antiproton-Ion Research) and Helmholtz Graduate School for Hadron and Ion Research (HGS-Hire).

[1] A. Hershcovitch, High-pressure arcs as vacuum-atmosphere interface and plasma lens for nonvacuum electron beam welding machines, electron beam melting, and nonvacuum ion material modification, J. Appl. Phys. 78, 5283 (1995).

[2] H. Maecker, Ein zylindrischer Bogen für hohe Leistungen, Z. Naturforsch. Teil A 11, 457 (1956).

[3] S. Namba, T. Endo, S. Fujino, C. Suzuki, and N. Tamura, Development of a cascade arc discharge source for an atmosphere-vacuum interface device, Rev. Sci. Instrum. 87, 083503 (2016).

[4] B.-L. Shi, S. Huang, K. Zhu, and Y.-R. Lu, Experimental study of the plasma window, Chin. Phys. C 38, 018201 (2014).

[5] S. Huang, K. Zhu, Y. R. Lu, S. Z. Wang, A. Hershcovitch, L. Yang, and X. Y. Zhang, Quantitative characterization of arc discharge as vacuum interface, Phys. Plasmas 21, 123511 (2014).

[6] H. Kuboki, H. Okuno, A. Hershcovitch, T. Dantsuka, H. Hasebe, K. Ikegami, H. Imao, O. Kamigaito, M. Kase, T. Maie, T. Nakagawa, and Y. Yano, Development of Plasma Window for gas charge stripper at RIKEN RIBF, J. Radioanal. Nucl. Chem. 299, 1029 (2014).

[7] Y. E. Krasik, S. Gleizer, V. Gurovich, I. Kronhaus, A. Hershcovitch, P. Nozar, and C. Taliani, Plasma window characterization, J. Appl. Phys. 101, 053305 (2007).
[8] W. A. J. Vijvers, D. C. Schram, A. E. Shumack, N. J. L. Cardozo, J. Rapp, and G. J. van Rooij, Experimental and theoretical determination of the efficiency of a subatmospheric flowing high power cascaded arc hydrogen plasma source, Plasma Sources Sci. Technol. 19, 065016 (2010).

[9] A. Hershcovitch, A plasma window for vacuumatmosphere interface and focusing lens of sources for nonvacuum ion material modification (invited), Rev. Sci. Instrum. 69, 868 (1998).

[10] B. T. Pinkoski, I. Zacharia, A. Hershcovitch, E. D. Johnson, and D. P. Siddons, X-ray transmission through a plasma window, Rev. Sci. Instrum. 72, 1677 (2001).

[11] A. Hershcovitch and A. Team, Air boring and nonvacuum electron beam welding with a plasma window, Phys. Plasmas 12, 057102 (2005).

[12] A. Giuliani, I. Yao, B. Lagarde, S. Rey, J.-P. Duval, P. Rommeluere, F. Jamme, V. Rouam, F. Wein, C. D. Oliveira, M. Ros, A. Lestrade, K. Desjardins, J.-L. Giorgetta, O. Laprevote, C. Herbaux, and M. Refregiers, A differential pumping system to deliver windowless VUV photons at atmospheric pressure, J. Synchrotron Radiat. 18, 546 (2011).

[13] D. Salzmann, Atomic Physics in Hot Plasmas (Oxford University Press, New York, 1998).

[14] M. A. Gigosos, M. Á. González, and V. Cardeñoso, Computer simulated Balmer-alpha, -beta and -gamma Stark line profiles for non-equilibrium plasmas diagnostics, Spectrochimica Acta Part B: Atomic Spectroscopy 58, 1489 (2003).

[15] H.-J. Kunze, Introduction to Plasma Spectroscopy (Springer, Berlin Heidelberg, 2009).

[16] A. Kramida, Yu. Ralchenko, and J. Reader (NIST ASD Team), NIST Atomic Spectra Database (ver. 5.6.1), [Online]. Available: https://physics.nist.gov/asd [2018, November 20]. National Institute of Standards and Technology, Gaithersburg, MD. (2018).

[17] A. Fridman and L.A. Kennedy, Plasma Physics and Engineering (CRC Press, Boca Raton, 2011).

[18] M. I. Boulos, P. Fauchais, and E. Pfender, Thermal Plasmas (Springer, New York, 1994).

[19] Y. P. Raizer, Gas Discharge Physics (Springer-Verlag, Berlin, 1991).

[20] G. M. W. Kroesen, C. J. Timmermans, and D. C. Schram, Expanding plasma used for plasma deposition, Pure Appl. Chem. 60, 795 (1988)

[21] A. A. Fridman and L. A. Kennedy, Plasma Physics and Engineering (Taylor \& Francis Routledge, London, 2011). 